\title{
Lentibulariaceae from Serra dos Pireneus, Goiás, Brazil'
}

\author{
Nuiawa Coelho ${ }^{2}$, Vera Lúcia Gomes-Klein ${ }^{2}$ \& Marcos Vinicius Dantas-Queiroz ${ }^{3,4}$
}

\begin{abstract}
This study provides a floristic survey of Lentibulariaceae occurring in Serra dos Pireneus (SDP), state of Goiás, Brazil. The phytogeographic domain in which SDP is located is the Cerrado, with different vegetation, including floodplain grasslands (campos úmidos) where Lentibulariaceae usually occur. Samples were collected from 2007 to 2010 and herbarium samples were consulted as well. We recorded nine species of Lentibulariaceae: the genera Genlisea (2 spp.) and Utricularia (7 spp.). Descriptions, illustrations, photographs and identification keys are presented in this study.

Key words: carnivorous plants, Cerrado, floristic survey, Genlisea, Utricularia.

\section{Resumo}

Este estudo apresenta um levantamento florístico das Lentibulariaceae ocorrentes na Serra dos Pireneus (SDP), estado de Goiás, Brasil. O bioma da SDP é o Cerrado, onde diferentes fitofisionomias ocorrem, incluindo campos úmidos, ambientes onde as Lentibulariaceae geralmente habitam. Coletas dos espécimes foram realizadas entre 2007 e 2010 e amostras de herbários também foram consultadas. Nove espécies foram encontradas em Lentibulariaceae: dos gêneros Genlisea (2 spp.) e Utricularia (7 spp.). Descrições, ilustrações, fotografias e chaves de identificação para estas espécies são apresentadas neste estudo.

Palavras-chave: plantas carnívoras, Cerrado, florística, Genlisea, Utricularia.
\end{abstract}

\section{Introduction}

Lentibulariaceae Rich. is a monophyletic family, belonging to Lamiales order (Müller et al. 2004; Cieslak et al. 2005; APG IV 2016) with a worldwide distribution, comprised in three genera, Genlisea A. St.-Hil., Pinguila L., and Utricularia L. (Taylor 1989; Heywood et al. 2007). Considered as carnivorous plants, their usual habitats are humid environments with nitrogen-poor soils, with terrestrial, aquatic and even epiphytic specimens (Taylor 1989).

Specimens of Lentibulariaceae typically occur in Cerrado, the second largest phytogeographic domain of Brazil and a hotspot for conservation (Myers et al. 2000; Klink \& Machado 2005). This domain occurs at altitudes ranging from $300 \mathrm{~m}$ to over $1,600 \mathrm{~m}$, with several different vegetation, including waterlogged habitats, the most suitable sites for the occurrence of Lentibulariaceae specimens (Taylor 1989;
Ribeiro \& Walter 2008). Nevertheless, natural areas of Cerrado have decreased over the years, mainly due to agricultural expansion (Miziara \& Ferreira 2007). Therefore, the Cerrado biodiversity study is one of the most relevant topics on the agenda of conservation biology.

Taxonomic studies about Lentibulariaceae occuring in areas of Cerrado were conducted by Fromm-Trinta (1979, 1981, 1996, 2004), Mamede \& Corrêa (2002), Guarçoni et al. (2006), da Silva et al. (2011) and Souza \& Bove (2012), with new species of the family recently described by Bove (2008) and Souza \& Bove (2011). The Brazilian Flora Group reports ca. 82 species, distributed in all Brazilian domains, and the Cerrado is the one with the highest number of species (BFG 2015).

To improve the biological knowledge and the distribution of the Brazilian Lentibulariaceae, we intend to provide a species list from Serra dos Pireneus, an area of conserved Cerrado. Herein

\footnotetext{
${ }^{1}$ This paper is a result of the undergraduation's conclusion study of N. Coelho.

${ }^{2}$ Universidade Federal de Goiás, Inst. Ciências Biológicas, Lab. Morfologia e Taxonomia Vegetal, Campus Samambaia, C.P. 131, 74001-970, Goiânia, GO, Brazil.

${ }^{3}$ Universidade Estadual Paulista "Júlio de Mesquita Filho", Campus Rio Claro, Inst. Biociências, Depto. Ecologia. Av. 24-A, 13506-900, Rio Claro, SP, Brazil.

${ }^{4}$ Author for correspondence: marvin.danque@gmail.com
} 
we include a dichotomous key, diagnosis, pictures and illustrations of the Lentibulariaceae from Serra dos Pireneus.

\section{Material and Methods}

Serra dos Pireneus (SDP) is located among the municipalities of Pirenópolis, Cocalzinho de

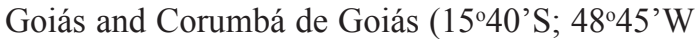
to $15^{\circ} 45^{\prime} \mathrm{S} ; 48^{\circ} 50^{\prime} \mathrm{W}$ ), in the state of Goiás (Fig. 1), with minimum altitude of $700 \mathrm{~m}$ in the municipality of Pirenópolis and maximum of $1,385 \mathrm{~m}$ on Pico dos Pireneus. Rock formations in sandstone and quartzite, dated from the Precambrian period constitute SDP's main features, which have several types of Cerrado phytophysiognomies, including seasonally floodplain grasslands and swampy areas (veredas) (Siqueira 2004). According to the Köpper-Geiger classification, the region climate is Aw type (Kottek et al. 2006), annual rainfall average is $1,535 \mathrm{~mm}$ and annual average temperature is 24 ${ }^{\circ} \mathrm{C}$ (Climate-Data.org 2016).

In the study area, there is the Parque Estadual da Serra dos Pireneus, created by Brazilian Law $10.321 / 87$, in order to "preserve the flora, fauna and springs therein, protecting natural sites of exceptional beauty and ensuring conditions of public welfare" (Goiás 1987). However, these protected areas are suffering many anthropogenic influences. There is an intense quartzite mining in SDP, destroying the organisms' natural habitats. Besides, the area is a touristic, folkloric and religious site, which enhance the flow of people and could compromise the ecologic interactions (Siqueira 2004; Bosquetti 2008).

Floristic surveys in SDP have been done since the eighteenth century (Pohl 1951; SaintHilaire 1975). Currently, projects about the regional flora of SDP are being conducted (e.g., Dantas-Queiroz et al. 2016) and this paper is part of this larger project.

Field trips were conducted in different months from 2007 to 2010 , in order to locate individuals, collect and observe them in their natural habitats. The specimens were deposited in UFG herbarium. Collections from CEN, HEPH, IBGE, NY, UB and UFG (acronyms according to Thiers, continuously updated) were also analyzed. Morphological and ecological information were extracted from collected materials. Additional materials, when necessary, were properly

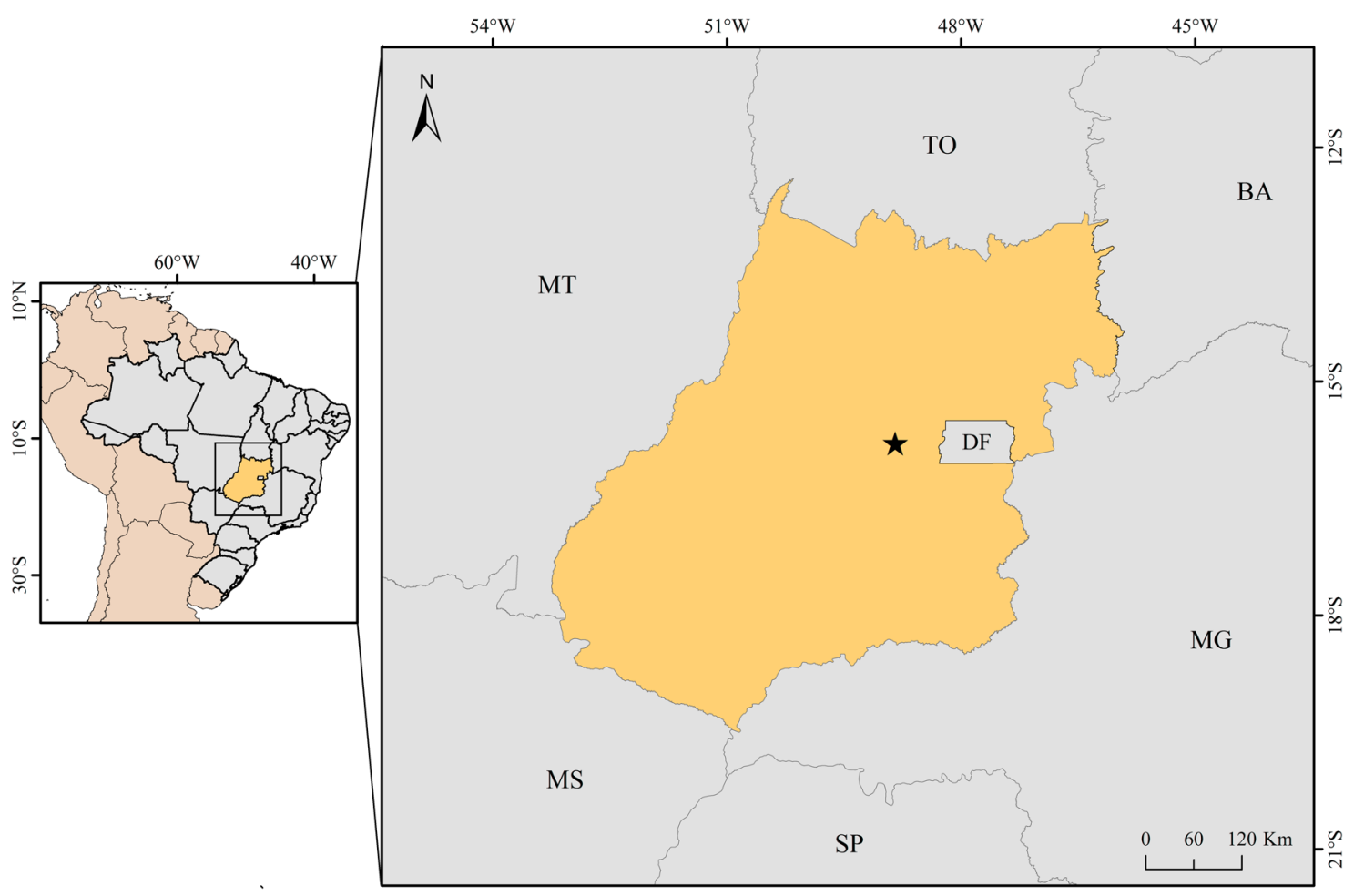

Figure 1 - Location of Serra dos Pireneus, in the state of Goiás, Brazil. 
referenced. The descriptions of the nomenclatural terms used were adapted from Taylor (1989) and Gonçalves \& Lorenzi (2011). A dichotomous key was prepared containing the largest number of floral reproductive characteristics, since vegetative characteristics do not always provide important information. A map was conceived using QGis Desktop 2.12.3, with shapefiles available at IBGE website (IBGE 2016).

\section{Results and Discussion}

There are nine species of Lentibulariaceae in SDP, distributed in two genera: Genlisea (two spp.) and Utricularia (seven spp.). This data represents ca. $20 \%$ of all Lentibulariaceae species from the state of Goiás (BFG 2015).

The species occur mostly on floodplain grasslands, sometimes growing over rocks (Utricularia neottioides A. St.-Hil. \& Girard). Many are associated with herbaceous vegetation, like Ceratophyllum L. (Ceratophyllaceae) and Poaceae, but also with other carnivorous plant, like Drosera L. (Droseraceae). The presence of specimens varies among the seasons, and individuals can be found with reproductive characters mostly during the rainy season. (October to May).

\section{Key to Lentibulariaceae species from Serra dos Pireneus}

1. Calyx 5-lobed; Y-shaped traps

1. Genlisea

2. Inflorescence 1-4 cm long simple; Corolla yellow; Flower 2-3 ............. 1.2. Genlisea pygmaea

2'. Inflorescence 10-40 cm long., Corolla purple; Flower 5-20.................1.1. Genlisea guianensis

1'. Calyx 2-lobed; traps as bladders

2. Utricularia

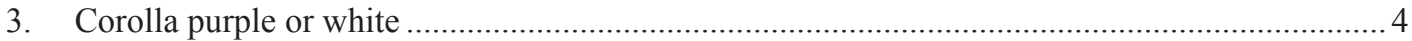

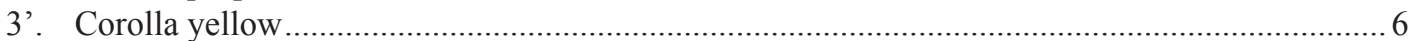

4. Corolla purple, rarely white, with a yellow spot; spur $1.5-2 \times$ longer than the corolla lower lip. 2.1. Utricularia amethystina

4'. Corolla white, lacking spots; spur the same size of the corolla lower lip............................. 5

5. Rheophytes, numerous individuals per population; calyx green or cream; capsule elliptical 2.4. Utricularia neottioides

5'. Geophytes, solitary individuals; calyx vinaceous; capsule globose

2.3. Utricularia hispida

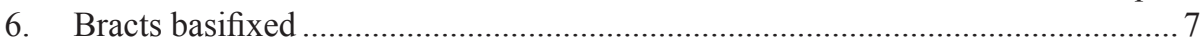

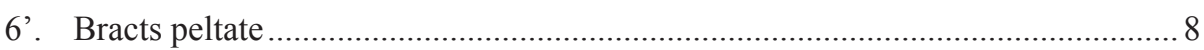

7. Terrestrial plants; bracts and sepals with a fimbriate margin ....

2.6. Utricularia simulans

7'. Aquatic plants; bracts and sepals with an entire margin

2.2. Utricularia gibba

8. Raceme flexuous; spur cylindrical, apex obtuse

2.7. Utricularia subulata

8'. Raceme erect; spur subulate, apex acute 2.5. Utricularia pusilla

1. Genlisea A. St.-Hil, Voy. Distr. Diam. 2: 428. 1833.

Rhizomatous herbs. Traps Y-shaped. Leaves aerial, rosulated, at base of flower stem, usually obovated or spatulated, with petiole. Inflorescence always racemose, rarely reduced to a single flower, erect, with or without bracts. Flowers yellow or purple, calyx persistent, 5-lobed, corolla 2-labiate, upper lip entire, lower lip curved, with spur; stamens 2, filaments curved, halteriform, anthers uniloculated; ovary globose, 2-carpels, 1-loculate, multiovulate, glabrous or trichomatous; style short, stigma 2-labiate. Fruit capsule, circumcised or longitudinal dehiscence. Seed numerous and small.

1.1. Genlisea guianensis N. E. Br., Icone. Pl. 27: t. 2629. 1900.

Figs. 2a,b; 5a

Annual or perennial herbs. Rhizoids filiform. Traps 1-8 cm long. Leaves not observed. Raceme 10-40 cm long., simple, slightly lignified; peduncle with sparse trichomes; bracts up to 1 $\mathrm{mm}$ long, lanceolate, trichomatous; several aborted 
reproductive branches. Flowers 5-20, rarely under 5; calyx 5-merous, upper sepals bigger than lower sepals, with sparse trichomes; corolla 7-15 mm long, purple, upper lip ovoid, apex 3-5 $\mathrm{mm}$ long, rounded, lower lip 4-10 $\mathrm{mm}$ long, 3 -lobed; spur subulate, apex 1.5-2 $\times$ higher than corolla, bifid. Capsules 1-2 mm long, globose, trichomatous.

Selected examined material: Pirenópolis, Serra dos Pireneus, 19.IV.2009, fl. and fr., M.V. Dantas-Queiroz et al. 19 (UFG); 15.V.2009, fl. and fr., N. Coelho et al. 117 (UFG).

Genlisea guianensis may differ from $G$. pygmaea for its long and slightly lignified raceme, bearing many purple flowers. Observed within water ponds. Observed flower period: April to May.

1.2. Genlisea pygmaea A. St.-Hil., Voy. Distr. Diam. 2: 431. 1833. Figs. 2c,d; 5b

Annual or perennial herbs. Rhizoids filiform. Traps 5-15 mm long. Leaves 5-10 $\times 0.5-1.5 \mathrm{~mm}$ long, forming dense rosettes, spatulate or narrowly rhombic, uninerveous, apex obtuse. Raceme 1-4 cm long, simple, peduncle with trichomes arranged in pairs; bracts up to $1 \mathrm{~mm}$ long, lanceolate, arranged in pairs, trichomatous; several aborted reproductive branches. Flowers 2-3, calyx 5-merous, sepals with trichomes; corolla 3-6 mm long, yellow, upper lip 1-3 mm long, apex rounded, lower lip 2-5 mm long, 3-lobed; spur subulate, $1.5 \times$ larger than the lower lip, apex acute. Capsule ca. $1 \mathrm{~mm}$ long, globose, trichomatous.

Selected examined material: Pirenópolis, Serra dos Pireneus, 10.IV.2009, fl., M.V. Dantas-Queiroz \& N. Coelho et al. 26 (UFG).

Additional examined material: Cristalina, Serra dos Topázios, 27.12.1972, fl. and fr., J.A. Rizzo 8714 (UFG); Alto Paraíso, Fazenda São Bento, fl., 23.II.1991, fl., D. Alvarenga et al. 765 (IBGE).

Genlisea pygmaea stands out for its tiny flower stem, with paired trichomes throughout its extension and the yellow flowers. It inhabits swamps, usually in sandy or clay soils between grasses. Observed flower period: December to April.
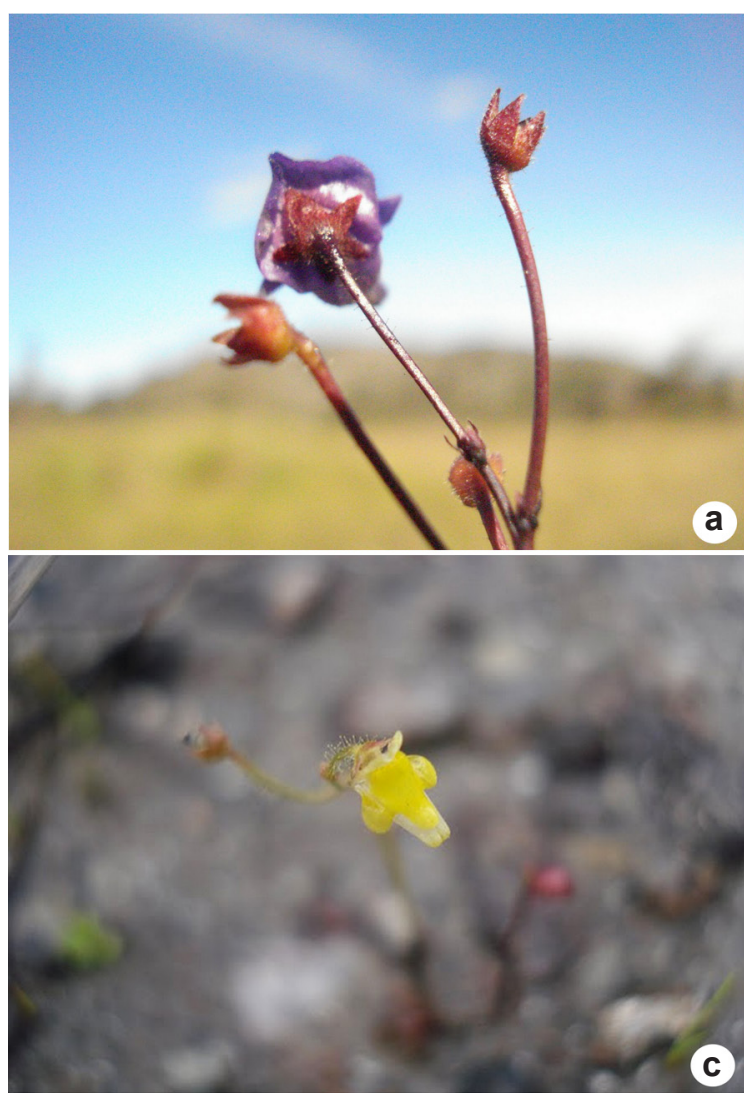

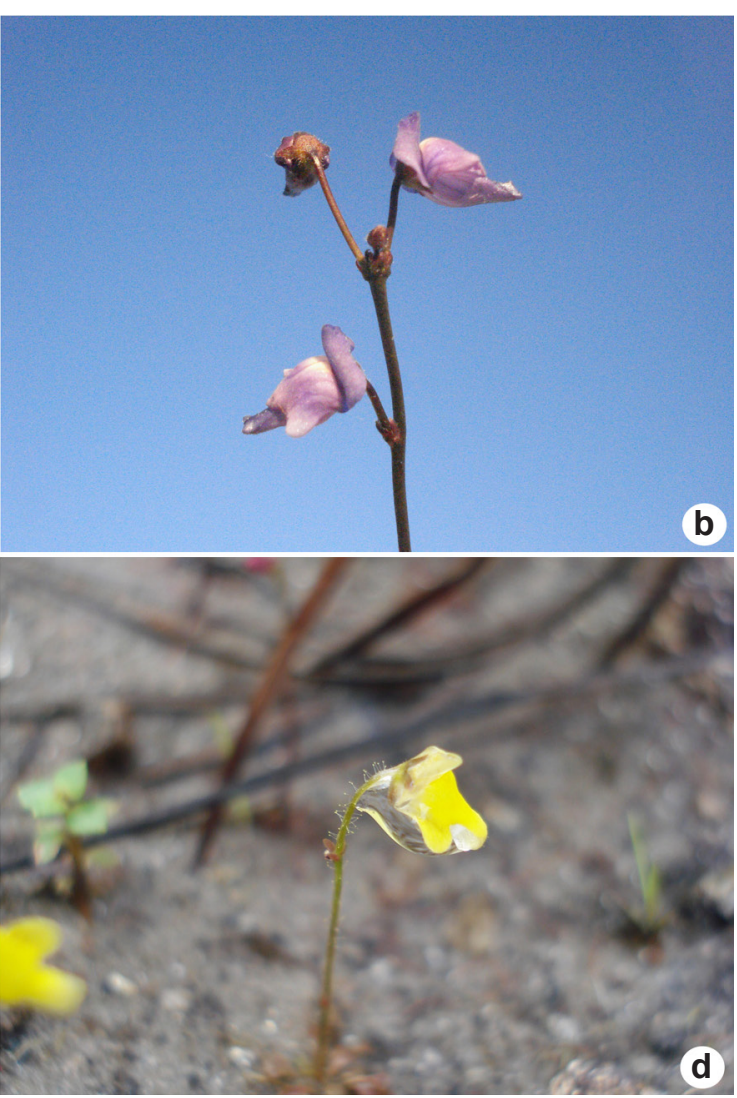

b

Figure 2 - Genlisea species from Serra dos Pireneus, GO, Brazil. a-b. G. pygmaea; c-d. G. guianensis. 
2. Utricularia L., Sp. Pl. 1: 18. 1753.

Aquatic or terrestrial herbs. Traps as an inserted utriculi (bladders) on vegetative parts, ovoid. Leaves petiolate, obovate, circular, filiform, sometimes dichotomously branched or even absent. Inflorescence racemose, rarely a single flower; bracts present, peduncle usually simple, unusually smaller than vegetative part, erect or flexuous, usually hairless, with scales (sterile bracts). Flowers yellow, white or purple; calyx 2-lobed, always persistent; corolla 2-labiate, papillose or glandular; upper lip entire, lower lip curved, entire or lobed, with spur; stamen 2, filaments curved, inserted on upper lip, anthers ellipsoidal; ovary globose or ovoid, 2-carpel, 1-locule, multiovulate, with or without hairs, style short, stigma 2-labiate. Fruit capsule, globose or ovoid; dehiscence dorsoventral, circumcised, longitudinal or indehiscent. Small and numerous seeds.

2.1. Utricularia amethystina Salzm. ex A. St.-Hil. \& Girard, Compt. Rend. Hebd. Séances Acad. Sci.7: 870. 1838.

Figs. 3a,b; 6c,d

Annual or perennial terrestrial herbs. Rhizoids similar to stolons. Stolons up to $10 \mathrm{~cm}$ long, filiform, branched. Traps ca. $1 \mathrm{~mm}$ long, along the stolon and rhizoid. Leaves 3-4 × 3-6 mm long, petiolate, obovate to circular (auricular), apex rounded. Raceme 6-27 cm long, simple, peduncle glabrous, erect; bracts ca. $1 \mathrm{~mm}$ long, basifixed, ovate, margins entire, apex acute; bracteoles smaller than the bracts; scales similar to the bracts. Flowers 1-4, pedicels 5-30 mm long, ascendant, filiform; calyx convex, margins entire, lobes unequal, glabrous, rarely with glandular trichomes, sometimes with a purple color; upper lobe 1.5-3 $\mathrm{mm}$ long, ovoid, apex rounded; lower lobe smaller, oblong-elliptical, apex emarginate; corolla 5-20 $\mathrm{mm}$ long, purple or white, upper lip elliptic, lower lip transversely obovate, usualy 3-lobed, with a yellow spot; spur 1.5-2 $\times$ longer than lower lip, curved. Capsule 1.5-2.5 mm long, globose.

Selected examined material: Pirenópolis, Serra dos Pireneus, 13.IX.2008, fl., N. Coelho et al. 104 (UFG); 18.IV.2009 (UFG), fl., M.V. Dantas-Queiroz et al. 13.

Additional material examined: Alto Paraíso, Chapada dos Veadeiros, fl. and fr., J.A. Rizzo 7940 (UFG); Alto Paraíso, Fazenda São Bento, 23.II.1991, fl. and fr., $D$. Alvarenga et al. 764 (IBGE).

Utricularia amethystina is easily found in SDP at the campos sujos (dense grassland), in rainy season. The corolla usually has a purple color and is scarcely observed in its white form. This natural color polymorphism was already reported by several authors (Taylor 1989; Silva et al. 2011; Baleeiro et al. 2016). Even so, U. amethystina is distinguished by its curved long spur and its yellow spot at the lower lip. Observed flower period: February to April and also in September.

\subsection{Utricularia gibba L., Sp. Pl. 1: 18. 1753.}

Figs. 4a; 5e

Aquatic herbs. Rhizoids not quite differentiated from stolons. Stolon filiform. Traps 1-2.5 mm long. Leaves 5-15 mm long, capillary, dichotomously branched, slighty flattened, apex setulose. Raceme 15-20 cm long, simple, peduncle glabrous, erect to flexuous; bracts ca. $1 \mathrm{~mm}$ long, basifixed, semicircular to peltate, margins entire, apex obtuse; bracteoles and scales absent. Flowers 1, pedicel 12-30 mm long, erect; calyx unequal, margins entire, sepals 1-3 mm long, apex rounded; corolla ca. $2 \mathrm{~mm}$ long, yellow, upper lip ovate, lower lip rounded; spur ca. $2 \mathrm{~mm}$. Capsule not observed.

Selected examined material: Corumbá de Goiás, Vale do Rio Corumbá, 17.I.1968, fl., H.S. Irwin 18722 (NY); Pirenópolis, Serra dos Pireneus, 13.IX.2008, fl., $N$. Coelho et al. 84 (UFG).

Utricularia gibba is an aquatic plant, scarcely observed in SDP, sometimes associated with G. guianensis within water ponds. The aquatic habit and the capillary dichotomously branched leaves can distinguish the species. Observed flower period: September.

2.3. Utricularia hispida Lam., Tabl. Encycl. 1: 50. 1791 Figs. $3 \mathrm{c} ; 5 \mathrm{f}$

Perennial terrestrial herbs. Rhizoids and stolon not observed. Traps $1-1.5 \mathrm{~mm}$ long. Leaves not observed. Raceme $20-80 \mathrm{~cm}$ long, simple, peduncle cylindrical, with trichomes at base, erect; bracts 1-2 mm long, basifixed, ovate, apex acute; scales numerous, similar to the bracts. Flowers 1-4, pedicel 10-30 mm, sinuous; calyx unequal, margins entire, sepals 1-3 mm, ovate, apex acute, vinaceous, lower lip smaller than upper lip; corolla 4-10 mm long, white, trichomatous, upper lip ovate-oblong, apex truncated; lower lip elliptical, 3-lobed; spur as long as lower lip, subulate, apex lanceolate Capsule 2-3 mm long, globose.

Selected examined material: Pirenópolis, Serra dos Pireneus, 15.X.2008, fl., N. Coelho et al. 111 (UFG); Additional material examined: Minaçu, Serra da Mesa, 9.XII.1991, fl. and fr, B.M.T. Walter et al. 873 (CEN). 

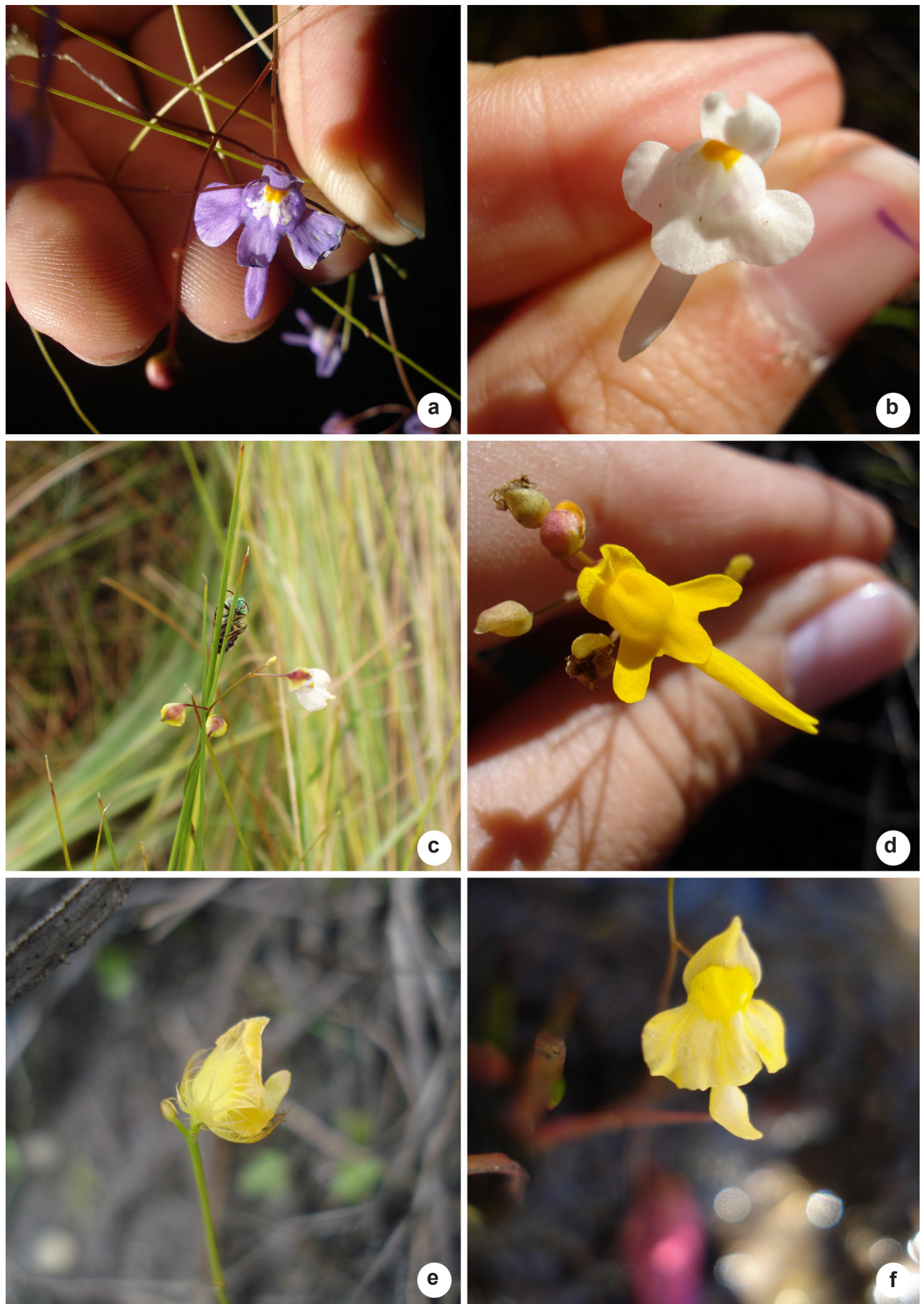

Figure 3 - Utricularia species from Serra dos Pireneus, GO, Brazil - a,b. U. amethystina - a. purple form; b. white form; c. U. hispida and an Euglossini bee; d. U. pusilla; e. U. simulans; f. U. subulata. 
Utricularia hispida is a robust species, noted by its tall raceme and the vinaceous sepals. This species is hard to sight, since the sympatric occurrence with tall grass in floodplain grasslands, covering almost the entire plant. An Euglossini bee was constantly observed on flowers, vibrating apparently to obtain some floral reward. Observed flower period: October to December.

2.4. Utricularia neottioides A. St.-Hil. \& Girard, Compt. Rend. Hebd. Séances Acad. Sci. 7: 869. 1838.

Figs. 4b; 5g

Perennial terrestrial (rupicolous) or rheophyte herbs. Rhizoids rigid, adhered to rocks. Stolon similar to rhizoids. Traps ca. $1 \mathrm{~mm}$ long. Leaves $3-50 \times 0.2-0,3 \mathrm{~mm}$, verticillate, filiform. Raceme 1-10(-32) cm long, simple, peduncule glabrous, erect; bracts $1-2 \mathrm{~mm}$ long, basifixed, ovate, margins entire, apex lanceolate; bracteoles absent; scales similar

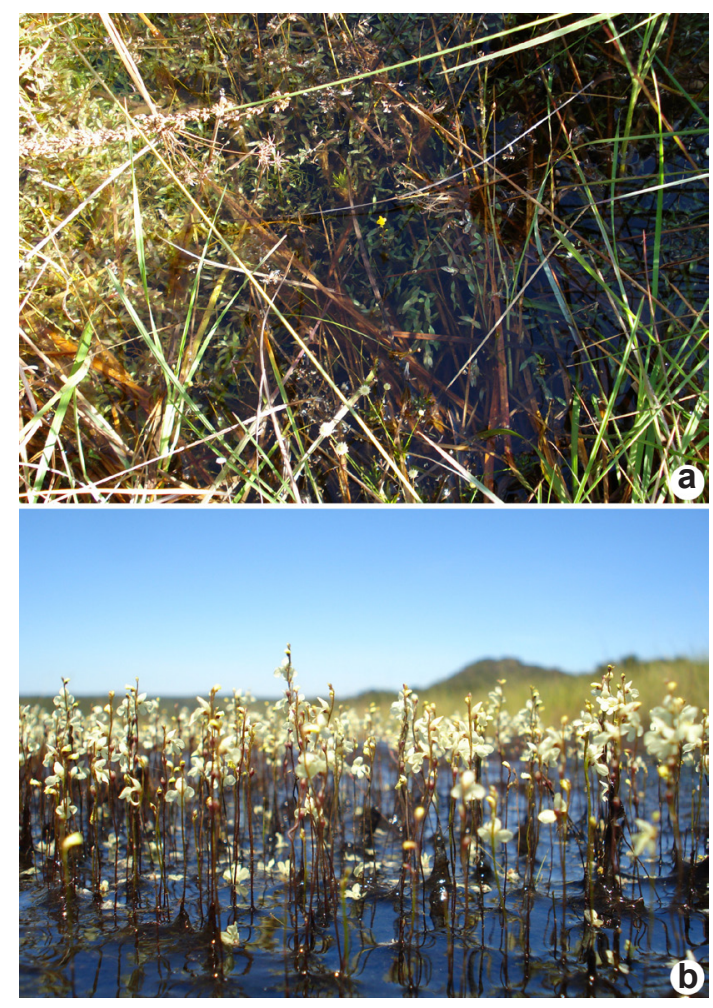

Figure 4 - Utricularia species from Serra dos Pireneus, GO, Brazil - a. a single U. gibba in a water pond; b. a population of $U$. neottioides, growing attached to the rocks. to bracts. Flowers $1-15$, pedicels $2-4 \mathrm{~mm}$ long; calyx 1-1.5 mm long, unequal, margins entire, green or cream; upper lobe long, apex lanceolate; lower lobe smaller than the upper, apex emarginate; corolla $1-4 \mathrm{~mm}$, cream or greenish; upper lip rounded, forming a hook, apex rounded; lower lip longer than the upper, conspicuous and 3-lobed; spur as long as the lower lip, curved upwards. Capsule $1-1.5 \mathrm{~mm}$ long, elliptical.

Selected examined material: Pirenópolis, Serra dos Pireneus, 4.III.2009, fl. and fr., M.V. Dantas-Queiroz et al. 9 (UFG); fl. and fr., $N$. Coelho et al. 112 (UFG); 16.IV.1994, fl. ande fr., $R$. César et al. 84 (UFG).

Additional material examined: Minaçu, Área de Empréstimo, ME 33, 10.III.1992, f1., T.B. Calvalcanti et al. 1127 (CEN).

Utricularia neottioides is usually found inside springs and close to slow waterfall courses, always adhered to rocks. This species is easily noticed by its populations, with large number of individuals aggregated, its filliform leaves and its rupicolous/reophyte habit. Observed flower period: March to April.

\subsection{Utricularia pusilla Vahl., Enum. Pl. 1: 202. 1804. \\ Figs. 3d; $5 \mathrm{~h}$}

Annual or perennial terrestrial herbs. Rhizoids and stolon filiform, branched. Traps ca. $1 \mathrm{~mm}$ long. Leaves not observed. Raceme 1-15 cm long, simple, peduncle glabrous, cilindrical, erect; bracts $1-2 \mathrm{~mm}$ long, peltate, ovate, margins entire, apex rounded; bracteoles absent; scales numerous, ovate. Flower 2-15, pedicels $2-4 \mathrm{~mm}$ long and erect; calyx unequal, margins entire, sepals $1-1.5 \mathrm{~mm}$ long, ovate; corolla 5-8 mm long, yellow, upper lip ovate, apex rounded; lower lip deeply 3-lobed; spur subulate, acute apex. Capsule 1-2 mm long, globose.

Selected examined material: Pirenópolis, Serra dos Pireneus, 19.IV.2008, fl., M.V. Dantas-Queiroz et al. 13 (UFG); 2008, fr., N. Coelho 113 (UFG); 10.VI.2009, fl. and fr., N. Coelho 114 (UFG); 13.VI.2009, N. Coelho 115 (UFG); 31.III.1990, fl. and fr., A.H. Salles et al. 1635 (HEPH).

Additional material examined: Uruaçu, Fazenda Pindaíba, 5.X.1994, fl. e fr., B.M.T. Walter et al. 2007 (CEN).

Utricularia pusilla is distinguished by its ovate sepals. Growing on sandy soils, in floodplain grasslands and on the watercourses margins. Observed flower period: March to June, and in October. 

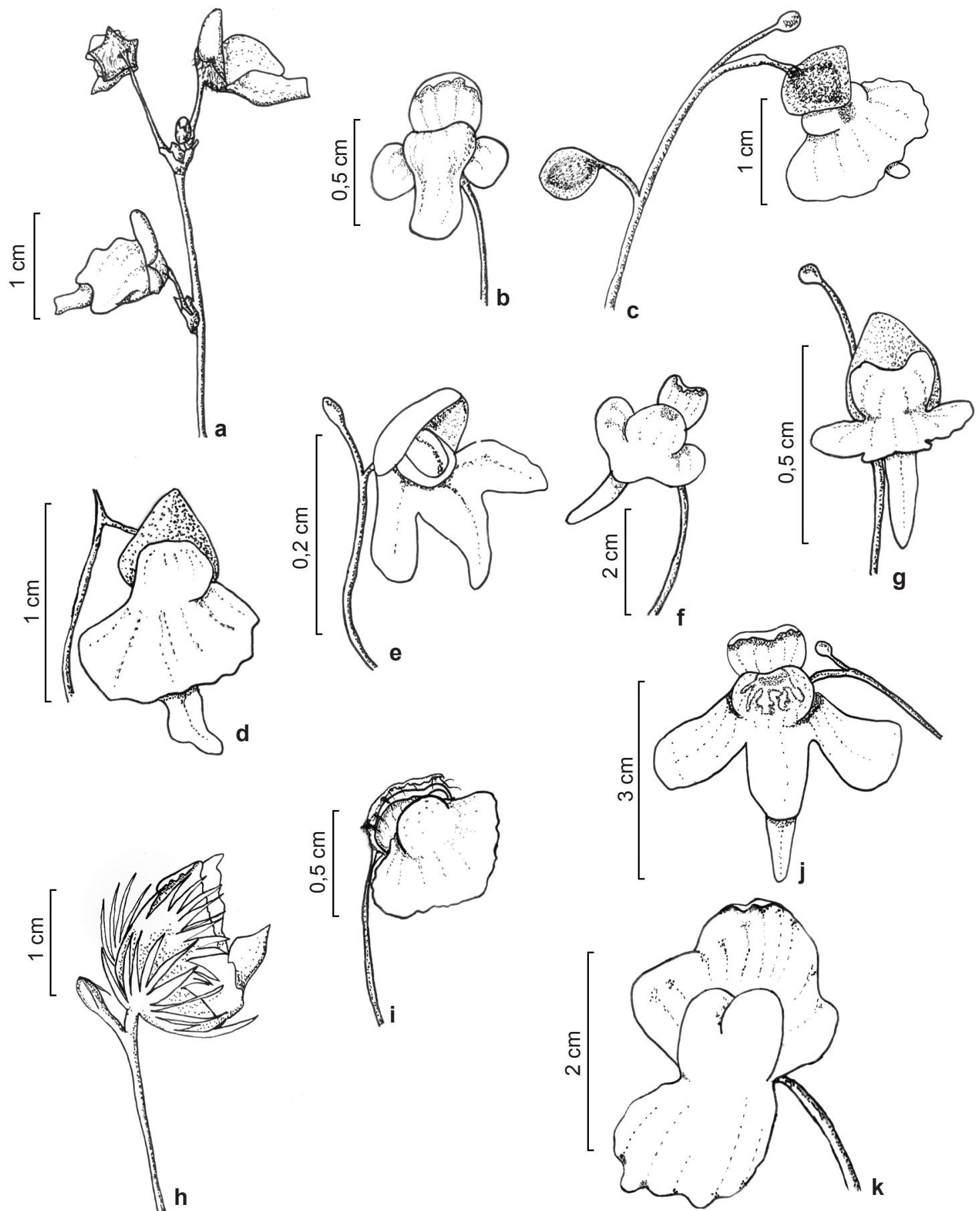

Figure 5 - Lentibulariaceae species from Serra dos Pireneus, GO, Brazil - a. Genlisea guianensis; b. G. pygmaea; c. Utricularia amethystina (purple form); d. U. amethystina (white form); e. U. gibba; f. U. hispida; g. U. neottioides; h. U. pusilla; i,j. U. simulans; k. U. subulata. 
2.6. Utricularia simulans Pilger, Notizbl. Königl. Bot. Gart. Berlin 6(56): 189. 1914.

Figs. 3e; 5i,j

Probably perennial terrestrial herbs. Rhizoids up to $1 \mathrm{~cm}$ long. capillary. Stolon similar to rhizoids. Traps ca. $1 \mathrm{~mm}$ long, adhered to stolon. Leaves not observed. Raceme $2.5-15 \mathrm{~cm}$ long, simple, peduncle glabrous, cylindrical, flexuous; bracts 1-2 mm long, basifixed, ovate, margin and apex fimbriated; bracteoles ca. $1 \mathrm{~mm}$ long, rounded, fimbriated; scales numerous, similar to the bracts. Flowers 2-5, congested, pedicels $1 \mathrm{~mm}$ long; calyx unequal, almost rounded, margins fimbriated, lower lobe longer than the upper, reddish; corolla 4-10 $\mathrm{mm}$ long, yellow, upper lip ovate, apex acute; lower lip rounded; spur as long or smaller than the lower lip, conical, apex acute. Capsule 2-5 mm long, globose.

Selected examined material: Pirenópolis, Serra dos Pireneus, 25.III.2009, fl., M.V. Dantas-Queiroz et al. 12 (UFG); 18.IV.2009, bt., M.V. Dantas-Queiroz 14 (UFG); 19.IV.2009, fl., M.V. Dantas-Queiroz 15 (UFG); 19.IV.2009, M.V. Dantas-Queiroz 16 (UFG); 15.V.2010, fl. and fr., $N$. Coelho 116 (UFG); 15.II.1973, fl. and fr., J.A. Rizzo et al. 8793 (UFG); Cocalzinho, Serra dos Pireneus, 14.III.2002, fl., M.L. Fonseca et al. 3223 (IBGE).

Utricularia simulans is noted by its fimbriate bracts and scales. Similar to U. fimbriata, (hence, 'simulans'), a species not found in Goiás state, with a fractiflex peduncle instead of an unarticulated peduncle in U. simulans (Taylor 1989). Observed growing in floodplain grasslands, with sandy and clay soils, sometimes associated with U. pusilla and $U$. subulata. Observed flower period: February to May.

\subsection{Utricularia subulata L., Sp. Pl. 1: 18. 1753.}

Figs. 3f; 5k

Annual or perennial terrestrial herbs. Rhizoids up to $1 \mathrm{~cm}$ long filliform. Stolon filliform, branched. Traps ca. $1 \mathrm{~mm}$ long, adhered to stolon. Leaves not observed. Raceme 3,5-30 cm long, simple, rarely branched, peduncle glabrous on apex and glandular at the bottom, flexuous; bracts $1 \mathrm{~mm}$ long, peltate, rounded, margins entire, apex rounded; bracteoles absent; scales numerous, similar to the bracts. Flowers 1-15, pedicel 5-10 mm long; calyx unequal, margins entire, sepals $1-1.5 \mathrm{~mm}$ long, concave, apex circular; corolla 5-10 mm long, yellow, upper lip ovate, apex rounded; lower lip rhombic to cuneate, slightly 3-lobed; spur slightly larger or the same size as the lower lip, cylindrical, apex obtuse. Capsule 1-1.5 mm long, globose.
Selected examined material: Pirenópolis, Serra dos Pireneus, 3.IV.2009, bt. and f1., N. Coelho et al. 117 (UFG).

Additional material examined: BRAZIL. DISTRITO FEDERAL: Samambaia, Parque Boca da Mata, 12.I.1996, fl. and fr., J.M. Rezende et al. 296 (CEN).

Utricularia subulata may be distinguished by its flexuous inflorescence, the rhombic to cuneate lower lip and its cylindrical spur. Occurring on wet fields, with sandy and clay soils. May occur associated with $U$. pusilla. Observed flower period: January to April.

\section{Acknowledgments}

The authors thank CNPq for the grant received for the project "Floristic survey of Serra dos Pireneus" and also thank Fundação de Amparo à Pesquisa do Estado de Goiás (FAPEG); Dra. Sirlene Aparecida Felisberto (in memorian) (UFG); Msc. Maria Aparecida da Silva from IBGE-DF; Dr. Climbiê Ferreira Hall, Dr. Augusto Francener, Msc. Alice Francener, Msc. Rodolph Delfino Sartin and Lia Wentzel for the help in the fieldtrips and shared knowledge that contributed for the writing of this paper; and Carmen Helena Monteiro (UFG) and the other herbarium curators that opened their collections.

\section{References}

APG IV - Angiosperm Phylogeny Group (2016) An update of the Angiosperm Phylogeny Group classification for the orders and families of flowering plants. Botanical Journal of the Linnean Society 181: 1-20.

Baleeiro PC, Jobson RW \& Sano PT (2016) Morphometric approach to address taxonomic problems: The case of Utricularia sect. Foliosa (Lentibulariaceae). Journal of Systematics and Evolution 54: 175-186.

BFG - The Brazil Flora Group (2015) Growing knowledge: an overview of seed plant diversity in Brazil. Rodriguésia 66: 1085-1113.

Bosquetti LDB (2008) Análise da estrutura da paisagem e fitofisionomias do Parque Estadual dos Pireneus, Goiás, Brasil. Tese de Doutorado. Universidade de São Paulo, Piracicaba. 134p.

Bove CP (2008) A new species of Utricularia (Lentibulariaceae) from central Brazil. Revista Brasileira de Botânica 31: 555-558.

Cieslak T, Polepalli JS, White A, Müller K, Borsch T, Barthlott W, Steiger J, Marchant A \& Legendre L (2005) Phylogenetic analysis of Pinguicula (Lentibulariaceae): Chloroplast DNA sequences and morphology support several geographically distinct radiations. American Journal of Botany 92: 1723-1736. 
Climate-Data.org (2015) Clima: Pirenopólis. Climate Data.org. Available at $<$ http://pt.climate-data.org/ location/43190/>. Access on 13 May 2016.

Dantas-Queiroz MV, Coelho N \& Gomes-Klein VL (2016) Iridaceae from Serra dos Pireneus, Goiás, Brazil. Nr. 3. Article 1902. Check List 12: 1-10.

Fromm-Trinta E (1979) Revisão das espécies do gênero Genlisea St.-Hil. Lentibulariaceae das regiões sudeste e sul do Brasil. Rodriguésia 31: 17-137.

Fromm-Trinta E (1981) Revisão do gênero Genlisea St.Hil. no Brasil. Boletim do Museu Nacional 61: 1-29.

Fromm-Trinta E (1996) Flora da Serra do Cipó, Minas Gerais: Lentibulariaceae. Boletim de Botânica da Universidade de São Paulo 15: 105-118.

Fromm-Trinta E (2004) Flora de Grão-Mogol, Minas Gerais: Lentibulariaceae. Boletim de Botânica da Universidade de São Paulo 22: 267-271.

Gonçalves EG \& Lorenzi H (2011) Morfologia vegetal: organografia e dicionário ilustrado de morfologia das plantas vasculares. Instituto Plantarum de Estudos da Flora, São Paulo. 544p.

Governo do estado de Goiás (1987) Lei no 10.321, de 20 de novembro de 1987. Legislação do estado de Goiás. Available at <http://www.gabinetecivil. goias.gov.br/leis_ordinarias/1987/lei_10321.htm>. Access on 13 May 2016.

Guarçoni EAE, Araújo RS \& Sartori MA(2006) Genlisea A. St.-Hil. Lentibulariaceae no Parque Estadual Serra do Cabral, Minas Gerais, Brasil. Sitientibus (série Ciências Biológicas) 7: 388-392.

Heywood VH, Brummitt RK, Culham A \& Seberg O (2007) Flowering plants families of the World. Royal Botanical Gardens, Kew, Richmond. 424p.

IBGE - Instituto Brasileiro de Geografia e Estatística (2016) Bases e referências cartográficas. Available at $<$ http://mapas.ibge.gov.br/bases-e-referenciais $>$. Access on 26 August 2016.

Klink CA \& Machado RB (2005) Conservation of the Brazilian Cerrado. Conservation Biology 19: 707-713.

Kottek M, Grieser J, Beck C, Rudolf B \& Rubel F (2006) World map of the Köppen-Geiger climate classification updated. Meteorologische Zeitschrift 15: 259-263.

Mamede MCH \& Corrêa MA (2002) Lentibulariaceae. In: Wanderley MG, Shepherd GJ, Giulietti AM, Melhem TS, Volker B \& Kameyama C (org.) Flora Fanerogâmica do estado de São Paulo. Instituto de Botânica, São Paulo. Vol. 2, pp. 141-154.
Miziara F \& Ferreira NC (2007) Expansão da fronteira agrícola e evolução da ocupação e uso do espaço no estado de Goiás: subsídios à política ambiental. In: Ferreira LG (ed.) A encruzilhada socioambiental - biodiversidade, economia e sustentabilidade no Cerrado. Editora UFG, Goiânia. Pp. 101-120.

Müller K, Borsch T, Legendre L, Porembski S, Theisen I \& Barthlott W (2004) Evolution of carnivory in Lentibulariaceae and the Lamiales. Plant Biology 6: 477-90.

Myers N, Mittermeier RA, Mittermeier CG, da Fonseca GAB \& Kent J (2000) Biodiversity hotspots for conservation priorities. Nature 403: 853-858.

Pohl JBE (1951) Viagem no interior do Brasil, empreendida nos anos de 1817 a 1821 e publicada por ordem de Sua Majestade o imperador da Áustria Francisco Primeiro. Instituto Nacional do Livro, Rio de Janeiro. 417p.

Ribeiro JF \& Walter BMT (2008) As principais fitofisionomias do bioma Cerrado. In: Sano SM, de Almeida SP \& Ribeiro JF (org.) Cerrado: ecologia e flora. Embrapa Cerrados / Embrapa Informação Tecnológica, Brasília. Pp. 153-154.

Rocha GF, Ferreira LG, Ferreira NC \& Ferreira ME (2011) Detecção de desmatamentos no bioma Cerrado entre 2002 e 2009: padrões, tendências e impactos. Revista Brasileira de Cartografia 63: 341-349.

Saint-Hilaire AFCP (1975) Viagem à província de Goiás. Universidade de São Paulo, São Paulo. 158p.

Silva NG, Alves RJV, Pereira JF \& Rivadavia F (2011) Lentibulariaceae, Serra de São José, Minas Gerais, Brazil. Check List 7: 120-127.

Siqueira JC (2004) Pirenópolis: identidade territorial e biodiversidade. Loyola, Rio de Janeiro. 79p.

Souza PCB \& Bove CP (2011) A new species of Utricularia (Lentibulariaceae) from Chapada dos Veadeiros (Central Brazil). Systematic Botany 36: 465-469.

Souza PCB \& Bove CP (2012) Flora dos estados de Goiás e Tocantins - Lentibulariaceae. Editora UFG, Goiânia. 69p.

Taylor P (1989) The genus Utricularia - a taxonomic monograph. Kew Bulletin Additional Series 14: $1-724$.

Thiers B [continuously updated] Index Herbariorum: A global directory of public herbaria and associated staff. New York Botanical Garden's Virtual Herbarium. Available at $<$ http://sweetgum.nybg. org/ih/>. Access on 13 May 2016. 